\title{
Plasma Fibrinogen Correlates with Metastasis and is Associated with Prognosis in Human Nasopharyngeal Carcinoma
}

\author{
Sha-Sha He ${ }^{1,2,3^{*}}$, Yan Wang1,2,3*, Lin Yang ${ }^{1,2,3}$, Hai-Yang Chen ${ }^{4}$, Shao-Bo Liang 5 , Li-Xia Lu1,2,3凶, \\ Yong Chen ${ }^{1,2,3} \bowtie$ \\ 1. Sun Yat-sen University Cancer Center, 651 Dongfeng Road East, Guangzhou, China. \\ 2. State Key Laboratory of Oncology in Southern China, Guangzhou, China. \\ 3. Collaborative Innovation Center for Cancer Medicine, Guangzhou, China. \\ 4. The Sixth Affiliated Hospital of Sun Yat-sen University, Guangzhou, China. \\ 5. The First Hospital of Foshan, Foshan, China. \\ "These authors contributed equally to this work.
}

$\triangle$ Corresponding authors: Yong Chen, M.D. Lixia Lu, M.D. PhD. Sun Yat-sen University Cancer Center, State Key Laboratory of Oncology in South China; Collaborative Innovation Center for Cancer Medicine, Department of Radiation Oncology, Guangzhou 510060, People's Republic of China. Telephone: +0086-020-87343505, E-mail: chenyong@sysucc.org.cn; lulx@sysucc.org.cn.

(c) Ivyspring International Publisher. This is an open access article distributed under the terms of the Creative Commons Attribution (CC BY-NC) license (https://creativecommons.org/licenses/by-nc/4.0/). See http://ivyspring.com/terms for full terms and conditions.

Received: 2016.07.29; Accepted: 2016.10.29; Published: 2017.02.10

\begin{abstract}
Background: The purpose of this observational study was to evaluate the prognostic significance of the pre-treatment plasma fibrinogen level for survival outcomes in nasopharyngeal carcinoma (NPC).

Methods: A total of 998 patients with NPC treated at a single centre in China were retrospectively enrolled, of whom 182 (18.2\%) developed distant metastasis during follow-up. Survival analyses were performed by the Kaplan-Meier method and Cox regression modelling to measure 3-year overall survival (OS) and distant metastasis-free survival (DMFS).

Results: Median OS for the entire cohort was 37.8 months. Using the cut-off value of $3.345 \mathrm{~g} / \mathrm{L}$ identified in receiver operating curve analysis for fibrinogen, a high pre-treatment plasma fibrinogen level were associated with older age $(P=0.034)$, advanced TNM stage $(P=0.004)$ and development of distant metastasis $(P<0.001$; Chi-square test). Multivariate Cox proportional hazard analysis demonstrated the pre-treatment plasma fibrinogen level was an independent significant prognostic factor for OS and DMFS in both the entire cohort and also among patients who developed distant metastasis during follow-up.

Conclusions: This study suggests the pre-treatment plasma fibrinogen level may serve as an independent prognostic marker to predict the survival outcomes of patients with NPC, including patients with metastatic disease.
\end{abstract}

Key words: Nasopharyngeal carcinoma; metastasis; fibrinogen; prognosis.

\section{Introduction}

Nasopharyngeal carcinoma (NPC) is endemic among the populations of Southern China and Southeast Asia; these regions have the highest prevalence of NPC worldwide [1, 2]. It was recently reported that NPC is related to the geographical district, age, gender, diet, lifestyle, Epstein-Barr virus
(EBV) infection, genetic factors, and even the type of dialect in China [3].

The therapeutic options for NPC have recently improved. Surgery is not usually possible because of the complex anatomical localization; however, NPC is sensitive to radiotherapy and chemotherapy. Due to 
the dose superiority of intensity-modulated radiotherapy (IMRT) compared to 2-dimensional radiotherapy (2D-RT), excellent locoregional control can now be achieved in NPC, even in locoregionally advanced disease, with a relatively low incidence of severe complications [4]. Consequently, IMRT has replaced 2D-RT as the primary treatment for non-disseminated NPC.IMRT alone is applied as a radical treatment for early stage NPC, whereas concurrent chemoradiotherapy (CCRT) with or without neoadjuvant chemotherapy (NACT) or adjuvant chemotherapy (ACT) is suggested for locoregionally advanced NPC in the National Comprehensive Cancer Network (NCCN) guideline.

As a result of the above-mentioned therapeutic improvements, the mortality rates for NPC peaked at 1.45/105 cases in the year 2002 and have decreased gradually since [5]. However, the improvements in locoregional control have not been accompanied by an increase in long-term overall survival. Moreover, in patients receiving IMRT, distant metastasis (DM) has become the predominant failure pattern [6-9].

The current TNM staging system for NPC currently possesses a number of limitations as it does not reflect tumor heterogeneity, and patients with the same TNM stage exhibit different clinical behaviours and achieve varied treatment responses and outcomes [10]. Therefore, there is currently much interest in identifying prognostic markers that would improve patient discrimination and guide the design and selection of more effective therapeutic strategies. However the identification of appropriate biomarkers remains a complex challenge.

Accumulating evidence suggests that activation of the blood coagulation system affects tumor cells and is closely related to the development, growth and metastasis of cancer [11]. Coagulation abnormalities have been associated with poor survival in patients with cancer $[12,13]$. Of the numerous haemostatic factors, plasma fibrinogen has been reported to be synthesized and overexpressed in human tumor cells. Fibrinogen is a key glycoprotein that functions as a non-specific acute-phase reactant and plays roles in the coagulation pathway, clot formation and wound healing [14, 15]. High fibrinogen level has been associated with various human malignancies, including colon [16], pancreatic [17] and renal cancer [18]. However, the role of fibrinogen in NPC remains unclear, especially in patients with metastatic disease.

Therefore, the purpose of this study was to explore whether the pre-treatment plasma fibrinogen level has potential as a prognostic factor in NPC, and furthermore assess the prognostic value of pre-treatment plasma fibrinogen in patients who develop metastasis after treatment.

\section{Materials and Methods}

\section{Patients}

The principal eligibility criteria for this retrospective study were: (1) biopsy-proven, undifferentiated, non-keratinising squamous cell carcinoma; (2) no sign of metastasis at diagnosis and no history of previous anti-cancer therapy; (3) age $\geq 18$ years old; (4) adequate hematologic, renal and hepatic function, with no history of venous thrombosis or anticoagulation therapy during the 3 months before treatment; and (5) complete follow-up data. In total, 998 patients with newly-diagnosed, non-metastatic NPC treated at Sun Yat-sen University Cancer Center, China, between January 2010 and October 2013 were retrospectively analyzed. This study was approved by the Research Ethics Committee of Sun Yat-sen University Cancer Center. Informed consent was obtained from all patients.

\section{Clinical stage}

The routine staging work-up included a complete medical history, physical examination, pre-treatment serum biochemistry analyses, direct reoptic nasopharyngoscopy, nasopharyngeal and neck magnetic resonance imaging (MRI), abdominal ultrasound, chest radiography and entire body bone scans, with or without positron emission tomography-computed tomography (PET-CT). Patients were staged according to the $7^{\text {th }}$ edition of the International Union against Cancer/American Joint Committee on Cancer (UICC/AJCC) staging system for NPC.

\section{Treatment}

All patients received primary curative-intent treatment according to the NCCN guidelines [19]. Concurrent chemoradiotherapy with or without neoadjuvant chemotherapy or adjuvant chemotherapy was administered for advanced-stage NPC (stage III-IVB), radiotherapy was administered for early-stage NPC or the patients with advanced-stage disease in poor health who could not tolerate the combined therapy.

All patients received radical IMRT. The cumulative radiation doses prescribed were 66-72 Gy at 2.12-2.43 Gy/fractions to the gross tumour volume of the nasopharynx (GTVnx), 64-70 Gy to the positive cervical lymph nodes (GTVnd), 60-63 Gy to the high-risk clinical target volume (CTV1) and 54-56 Gy to the low-risk clinical target volume (CTV2). Radiotherapy was completed within approximately 7 weeks. The doses received by each organ at risk (OAR) were limited according to the dose tolerances of the RTOG 0225 protocol [20], with dose reductions 
applied as far as possible without sacrificing the tumor target coverage.

The most common first-line chemotherapy were platin-based strategies alone (cisplatin, carboplatin) or platins in association with docetaxel, paclitaxel, gemcitabine or capecitabine with or without 5-fluorouracil. Neoadjuvant chemotherapy was 80 $\mathrm{mg} / \mathrm{m}^{2}$ cisplatin on day 1 and $4.0 \mathrm{~g} / \mathrm{m}^{2} 5$-fluorouracil as a 72 hours continuous intravenous infusion every 3 weeks for two or three cycles. Concurrent chemotherapy consisted of intravenous cisplatin (40 $\mathrm{mg} / \mathrm{m}^{2} /$ day) given weekly or $\left(80 \mathrm{mg} / \mathrm{m}^{2} /\right.$ day) on weeks 1, 4 and 7 of radiotherapy.

In patients with metastasis at diagnosis, the main treatment modality was chemotherapy. Radiotherapy was always prescribed for localized metastatic lesions to cure or relieve pain; targeted therapy was also added for palliative purposes. The choice of therapy was individualized, based on each patient's characteristics including their performance status, treatment expectations, financial resources, etc.

\section{Follow-up}

Follow-up evaluations were performed every 3 months within the first 3 years, 6 months in the following 3-5 years, and annually thereafter until death. Each follow-up visit included a physical examination, nasopharyngoscopy, nasopharyngeal and neck magnetic resonance imaging (MRI), chest $\mathrm{CT}$, abdominal ultrasonography, bone marrow scan, and assessment of EBV DNA load and other haematological parameters.

Follow-up was measured from date of first diagnosis to either the date of death or loss to follow-up. Overall survival (OS) was defined as the time from first diagnosis to death or loss to follow-up or January, 2016. Distant metastasis-free survival (DMFS) was measured to first distant metastasis or censorship at last follow-up. The disease-free interval (DFI) was assessed as the time between the start of primary treatment and first diagnosis of metastasis.

\section{Statistical analysis}

Cut-off values for pre-treatment plasma fibrinogen and Epstein-Barr viral DNA (EBV DNA) load were established using receiver operating characteristic (ROC) analysis. The Chi-square test was used to analyze the associations between pre-treatment plasma fibrinogen and clinicopathological features. Univariate and multivariate analysis of clinical variables were performed using Cox proportional hazards regression models. Survival rates were plotted against time using the Kaplan-Meier method, and log-rank testing was used to assess the differences between curves.
Statistical analysis was performed using IBM SPSS statistical program package, version 22.0, IBM Corp. All statistical tests were two-sided; $P<0.05$ was defined as statistically significant.

\section{Results}

\section{Pre-treatment plasma fibrinogen is associated with the clinicopathological features of the disease}

The characteristics of the 998 patients included in this study are summarized in Table 1 . ROC analysis identified $3.345 \mathrm{~g} / \mathrm{L}$ as the cut-off value for pre-treatment plasma fibrinogen with respect to OS (AUC, 0.614; sensitivity, 0.798; specificity, 0.509). This cut-off value was employed in all subsequent analyses. In total, 655/998 (65.6\%) patients had a pre-treatment plasma fibrinogen level below 3.345 $\mathrm{g} / \mathrm{L}$ and 343/998 (34.4\%) had a pre-treatment plasma fibrinogen level equal to or greater than the cut-off value. A high pre-treatment plasma fibrinogen level was associated with older age $(P=0.034)$, advanced TNM stage $(P=0.004)$ and advanced T category $(P<$ 0.001). Moreover, patients who developed distant metastasis during follow-up had higher pre-treatment plasma fibrinogen levels than patients who did not develop distant metastasis during follow-up $(P<$ 0.001; Table 1).

Table 1. Summary of the clinicopathological features of the 998 patients with nasopharyngeal carcinoma.

\begin{tabular}{|c|c|c|c|c|}
\hline \multirow[t]{2}{*}{ Characteristic } & \multirow{2}{*}{$\begin{array}{l}\text { Total } \\
\text { (No, \%) }\end{array}$} & \multicolumn{2}{|c|}{ Fibrinogen (g/L) } & \multirow[t]{2}{*}{$P$-value ${ }^{*}$} \\
\hline & & $<3.345$ & $\geq 3.345$ & \\
\hline & $998(100)$ & $655(65.6)$ & $343(34.4)$ & \\
\hline Age (years) & & & & 0.034 \\
\hline$<46$ & $535(53.6)$ & $367(56)$ & $168(49)$ & \\
\hline$\geq 46$ & $463(46.4)$ & $288(54)$ & $175(51)$ & \\
\hline Smoking & & & & 0.260 \\
\hline Yes & $290(29.2)$ & $198(30.2)$ & $92(26.8)$ & \\
\hline No & $457(69.8)$ & $457(69.8)$ & $251(73.2)$ & \\
\hline Overall stage (7th edition) & & & & 0.004 \\
\hline $\mathrm{I}+\mathrm{II}$ & $113(11.3)$ & $88(13.4)$ & $25(7.3)$ & \\
\hline III+IV & $885(88.7)$ & $567(86.6)$ & 318 (92.7) & \\
\hline $\mathrm{T}$ category & & & & $<0.001$ \\
\hline $\mathrm{T} 1-2$ & $235(23.5)$ & $181(27.6)$ & $54(15.7)$ & \\
\hline T3-4 & $763(76.5)$ & $474(72.4)$ & $289(84.3)$ & \\
\hline $\mathrm{N}$ category & & & & 0.055 \\
\hline N0-1 & $452(45.3)$ & $311(47.5)$ & $141(41.1)$ & \\
\hline N2-3 & $546(54.7)$ & $344(52.5)$ & $202(58.9)$ & \\
\hline Treatment & & & & 0.978 \\
\hline IMRT & 209 (20.9) & $137(20.9)$ & $72(21)$ & \\
\hline CT + IMRT & $789(79.1)$ & $518(79.1)$ & $271(79)$ & \\
\hline $\begin{array}{l}\text { Post-treatment distant } \\
\text { metastasis }\end{array}$ & & & & $<0.001$ \\
\hline Yes & 219 (21.9) & $122(18.6)$ & $97(28.3)$ & \\
\hline No & 779 (78.1) & $533(81.4)$ & $246(71.7)$ & \\
\hline
\end{tabular}

Abbreviations: $\mathrm{IMRT}=$ intensity-modulated radiation therapy; $\mathrm{CT}=$

chemotherapy.

* Chi-square test. 
A

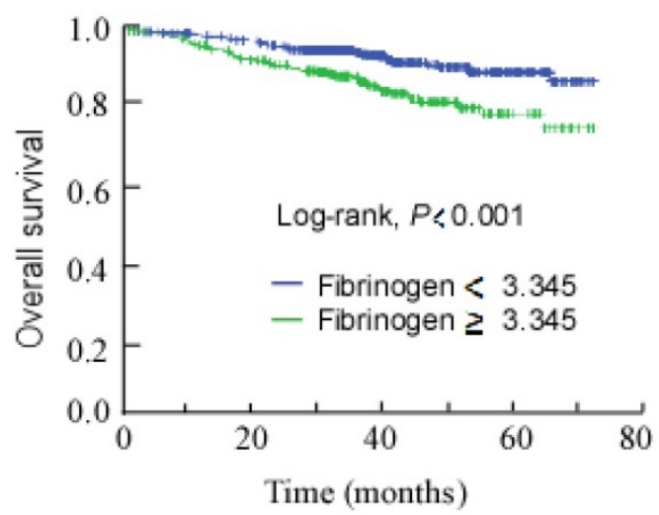

B

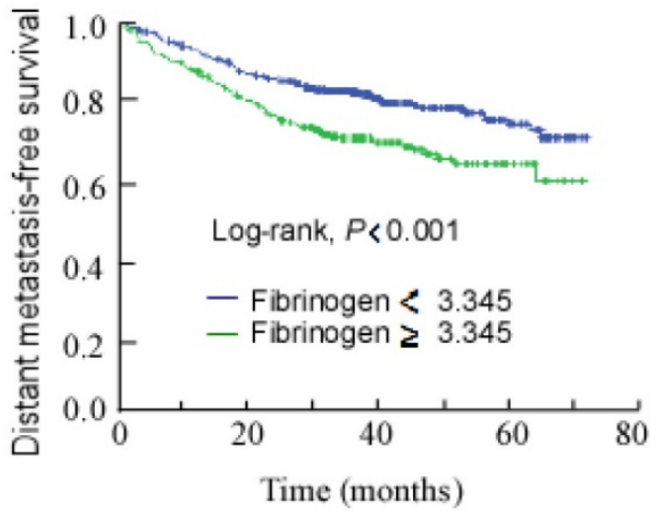

Figure 1. Kaplan-Meier overall survival (A) and distant metastasis-free survival (B) curves for all 998 patients with NPC stratified by pre-treatment plasma fibrinogen level $(\geq 3.345 \mathrm{~g} / \mathrm{L}$ vs. $<3.345 \mathrm{~g} / \mathrm{L})$.

\section{High pre-treatment plasma fibrinogen is an independent prognostic factor for poor OS and DMFS}

The median follow-up duration was 37.8 months (range, 0.07-73.7 months) for the entire cohort of 998 patients. The 3-year overall survival rate was $92.1 \%$. In survival analysis, a high pre-treatment plasma fibrinogen level was associated with poorer 3-year OS and DMFS than a low pre-treatment plasma fibrinogen level $(87.5 \%$ vs. $94.3 \%$ and $72.3 \%$ vs. $83.3 \%$; Fig. 1A and 1B; log-rank test, both $P<0.001$ ).

To determine the independent prognostic value of the plasma fibrinogen level for OS and DMFS, multivariate analyses using a Cox proportional hazard model were performed to adjust for known prognostic parameters including overall stage and EBV DNA load. The multivariate analyses demonstrated that a high pre-treatment plasma fibrinogen level was an independent prognostic factor for poor OS (HR 1.970, 95\% CI 1.324-2.931, $P=0.001$ ) and poor DMFS (HR 1.518, 95\% CI 1.159-1.989, $P=$ 0.002; Table 2).

Table 2. Multivariate analysis of prognostic factors in the 998 patients with nasopharyngeal carcinoma.

\begin{tabular}{lllll}
\hline Endpoint & Characteristic & HR & $95 \%$ CI & $P$-value* \\
\hline OS & Fibrinogen & 1.970 & $1.324-2.931$ & 0.001 \\
& EBV DNA & 1.923 & $1.290-2.868$ & 0.001 \\
DMFS & Fibrinogen & 1.518 & $1.159-1.989$ & 0.002 \\
& EBV DNA & 1.953 & $1.490-2.559$ & $<0.001$ \\
\hline
\end{tabular}

Abbreviations: OS = overall survival; DMFS = distant metastasis-free survival; $\mathrm{HR}$ $=$ hazard ratio; $\mathrm{CI}=$ confidence interval; *: Multivariate $P$-values were calculated using an adjusted Cox proportional hazards model.

\section{High pre-treatment plasma fibrinogen is associated with the development of distant metastasis after treatment}

In total, $182(18.2 \%)$ of the 998 patients with NPC developed distant metastasis after treatment (i.e. during follow-up), with the bones the most common site of metastasis. Overall, 166 (91.2\%) of these 182 patients developed metastasis within 3 years after treatment. The mean $( \pm S D)$ pre-treatment plasma fibrinogen level of patients were summarized in Table 3 , in non-metastatic patients, pre-treatment fibrinogen level was significantly lower than in patients with bone metastasis $(P<0.001)$, lung metastasis $(P<$ $0.001)$, liver metastasis $(P<0.001)$. In addition, there were no differences between bone, lung and liver groups of pre-treatment fibrinogen values (Table 3).

Table 3. Pre-treatment plasma fibrinogen levels of patients with different outcomes.

\begin{tabular}{lllc}
\hline Outcome & Total & \multicolumn{2}{l}{ Plasma fibrinogen level $(\mathrm{mg} / \mathrm{dl})$} \\
\cline { 2 - 4 } & $(\mathrm{No})$. & Median & Mean \pm SD \\
\hline Non-metastatic cases & 816 & 3.080 & $3.229 \pm 0.963$ \\
Distant metastasis & 182 & 3.140 & $3.353 \pm 1.068$ \\
Bone metastasis & 89 & 3.140 & $3.404 \pm 1.081$ \\
Lung metastasis & 71 & 3.260 & $3.534 \pm 1.217$ \\
Liver metastasis & 76 & 3.360 & $3.438 \pm 1.129$ \\
\hline
\end{tabular}

Abbreviations: SD $=$ Standard Deviation.

\section{High pre-treatment plasma fibrinogen is an independent prognostic factor for OS in metastatic patients}

Given its associations with OS and DMFS in the entire cohort, the associations between the pre-treatment plasma fibrinogen level and the survival outcomes of patients who developed distant metastasis after treatment were examined. 
Kaplan-Meier survival analyses revealed the low pre-treatment plasma fibrinogen $(<3.345 \mathrm{~g} / \mathrm{L})$ subgroup achieved better 3-year OS than the high pre-treatment plasma fibrinogen subgroup $(85.4 \%$ vs. $70.1 \%$; Fig. 2A; $P=0.016)$. Furthermore, high pre-treatment plasma fibrinogen was associated with significantly poorer 3-year OS among patients who developed bone metastasis $(48.8 \%$ vs. $72.3 \%$ for the low pre-treatment fibrinogen group; Fig. 2B; $P=$ $0.021)$, but not among the patients who developed lung or liver metastases (Fig. 2C and 2D).

After adjusting for the disease-free interval, the pre-treatment plasma fibrinogen level and pre-treatment plasma EBV DNA load were identified as significant prognostic factors for OS in univariate analysis. Multivariate analysis confirmed that pre-treatment plasma fibrinogen was an independent prognostic factor for OS (HR 2.384, 95\% CI $1.021-5.565, P=0.045$ ); the disease-free interval (HR 2.666, 95\% CI 1.577-4.509, $P<0.001)$ and pre-treatment plasma EBV DNA load (HR 1.702, 95\% CI 1.019-2.844, $P=0.042$ ) were also independent significant prognostic factors for OS.

\section{Discussion}

In this retrospective study, high pre-treatment plasma fibrinogen was found to be associated with advanced age, T category and TNM stage and distant

$\mathbf{A}$

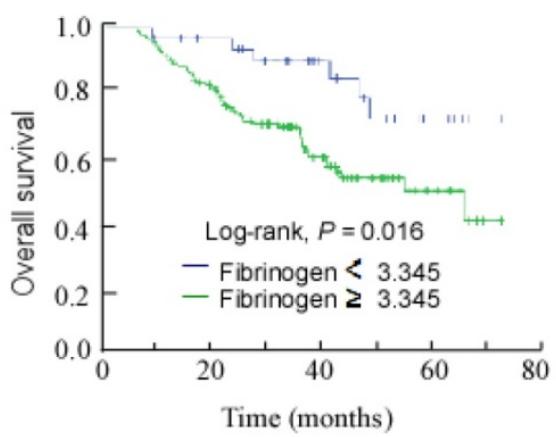

C

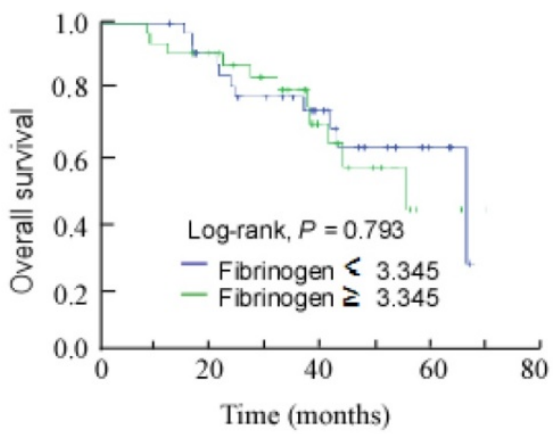

metastasis and poorer overall survival in NPC. Previous studies have demonstrated similar associations between fibrinogen and prognosis in other tumor types; however, there have been few studies to assess the prognostic value of pre-treatment plasma fibrinogen in NPC. Recently, Lan et al. [21] reported pre-treatment plasma fibrinogen and pre-treatment plasma EBV DNA had clinical significance in patients with stage IVA/B NPC treated with IMRT: higher pre-treatment plasma fibrinogen was associated with poorer disease-specific survival (DSS), disease-free survival (DFS) and DMFS. Moreover, high pre-treatment plasma fibrinogen had superior prognostic value to the pre-treatment plasma EBV DNA load [21], even though EBV DNA has been established as one of the most significant prognostic factors in NPC [22]. Another study reported that both high pre-treatment plasma EBV DNA and fibrinogen were associated with significantly pooer DFS, DMFS and OS [23]. In line with these studies, our multivariate analyses confirmed pre-treatment plasma fibrinogen was an independent prognostic factor for OS and DMFS in patients with NPC treated with IMRT. Moreover, pre-treatment plasma fibrinogen was also an independent prognostic factor for OS among the group of patients who developed metastasis after treatment.

\section{B}

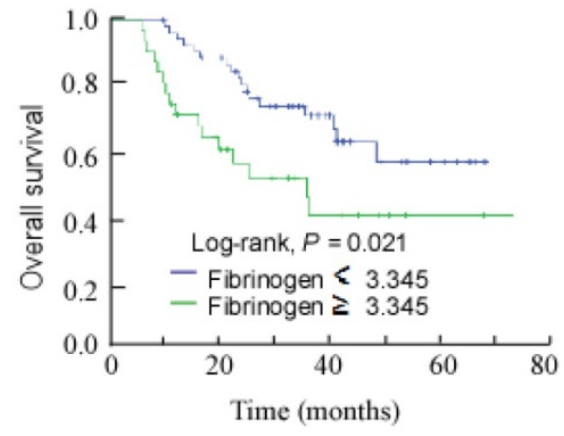

D

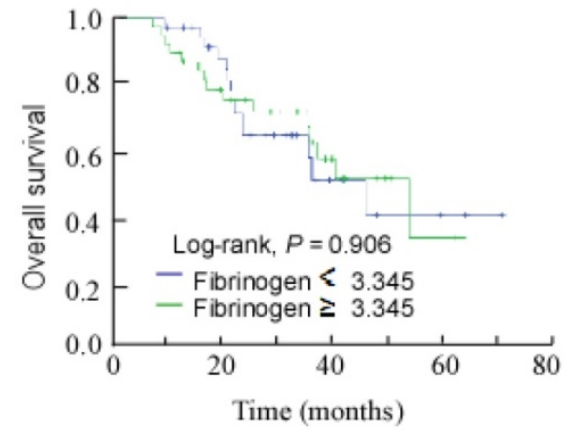

Figure 2. Kaplan-Meier overall survival curves for the patients who developed metastasis during follow-up stratified by pre-treatment plasma fibrinogen level ( $\geq$ $3.345 \mathrm{~g} / \mathrm{L}$ vs. < $3.345 \mathrm{~g} / \mathrm{L}$ ). (A) All patients with distant metastasis; (B) patients with bone metastasis; (C) patients with lung metastasis; (D) patients with liver metastasis. 
Our analysis indicates a high fibrinogen level is clinically-relevant in patients with advanced NPC. This finding is in accordance with previously published data on patients with other malignancies $[18,24,25]$. However, the pre-treatment plasma fibrinogen level was not associated with lymph node involvement in this cohort, further indicating that a high fibrinogen level is associated with haematogenous metastasis. Moreover, our analyses strongly suggest that suffering distant metastasis is more likely to have higher pre-treatment plasma fibrinogen level, while, the mesurement of fibrinogen during/after treatment is necessary to identify the role during tumor progression and metastasis in the future.

Gao et al. reported that an increased platelet count was associated with distant metastasis in NPC [26]. Fibrinogen is the predominant coagulation factor in blood plasma and promotes platelet aggregation and formation of fibrin [27]. Fibrin remodelling has been confirmed to play a crucial role in the formation of new vessels, which promotes metastasis [28]. Under the circumstances of hypercoagulability, fibrinogen is deposited in the extracellular matrix, binds to fibroblast growth factors or vascular endothelial cell growth factors, and thereby promotes cellular adhesion, proliferation and migration [29, 30]. These processes enable malignant cells to leave the primary tumor, migrate into the lymphovascular and circulatory systems, and establish a new blood supply in order to survive at the site of metastasis [31]. Verheul et al. [32] demonstrated that vascular endothelial cell growth factor (VEGF), a marker of angiogenesis, was expressed in all renal cell carcinoma (RCC) specimens examined using immunohistochemistry, and fibrinogen was detected close to blood vessels in $84 \%$ of these RCC specimens. More importantly, Verheul et al. reported that fibrinogen expression adjacent to tumor cells was independently related to VEGF expression in multivariate analysis. Thus, these data provide evidence of an interaction between angiogenesis and tumor cells and the presence of fibrinogen. On this basis, several groups have recently investigated the relationship between high plasma fibrinogen levels and the clinical features and survival of patients with cancer. The use of therapeutic anti-angiogenic agents has become a popular cancer treatment, and targeting VEGF has become an integral component of the treatment regimens for many types of cancer [33]. Therefore, Phase III studies involving large numbers of patients with adequate follow-up are required to confirm the association between plasma fibrinogen and the outcomes of patients with NPC, and also to determine whether plasma fibrinogen can be used to predict the response to anti-angiogenic therapy. Such studies would help to validate the prognostic value of fibrinogen in NPC, and may provide additional insight into potential therapeutic targets for NPC.

Although some of the mechanisms that link fibrinogen to metastasis have been identified [29, 30], the prevention and treatment of distant metastasis remains a complex challenge and, despite the application of aggressive treatment regimens, the survival outcomes of patients with distant metastasis are extremely poor [34]. In the current study, patients who developed distant metastasis achieved a median survival time of 13.9 months after diagnosis of distant metastasis, even when individualized therapy was administered. Therefore, preventing metastasis now represents a key strategy to improve the survival outcomes of patients with NPC.

We hope this study will provide a basis for the future direction of clinical strategies for NPC. Firstly, further studies are necessary to explore whether the threshold for initiating targeted interventions should be lowered for patients with a high pre-treatment plasma fibrinogen level, and to assess whether these patients benefit from anti-coagulative agents to reduce the risk of metastasis or thromboembolic events. Moreover, collecting plasma samples from patients and monitoring fibrinogen levels during follow-up in a longitudinal study would help to clarify the effect of treatment on the relationships between NPC and the coagulation system. Moreover, the pre-treatment plasma fibrinogen level could possibly be incorporated into prognostic systems and nomograms that combine independent factors to predict the prognosis of patients with NPC.

\section{Conclusions}

This study indicates elevated pre-treatment plasma fibrinogen is associated with advanced TNM stage and has significant prognostic value for distant metastasis after treatment and poorer OS in NPC. We suggest that pre-treatment plasma fibrinogen represents a useful tool for clinical decision-making, especially when selecting individualized treatment to prevent metastasis. Moreover, pre-treatment plasma fibrinogen was associated with a poorer prognosis among patients who developed metastasis after treatment; therefore, fibrinogen may have potential for predicting the response to anti-angiogenic therapy. However, further experimental and clinical investigations are warranted to confirm this hypothesis.

\section{Acknowledgments}

This work was supported by the Natural Science Foundation of Guangdong Province, China [grant 
number 2016A020215083]. The funding agency had no role in the study design, data collection and analysis, decision to publish, or preparation of the manuscript.

\section{Competing Interests}

The authors have declared that no competing interest exists.

\section{References}

1. Wee JT, Ha TC, Loong SL, Qian CN. Is nasopharyngeal cancer really a "Cantonese cancer"? Chinese journal of cancer. 2010; 29: 517-26.

2. Yu MC, Yuan JM. Epidemiology of nasopharyngeal carcinoma. Seminars in cancer biology. 2002; 12: 421-9.

3. Cao SM, Simons MJ, Qian CN. The prevalence and prevention of nasopharyngeal carcinoma in China. Chinese journal of cancer. 2011; 30: 114-9.

4. Xiao WW, Huang SM, Han F, Wu SX, Lu LX, Lin CG, et al. Local control, survival, and late toxicities of locally advanced nasopharyngeal carcinoma treated by simultaneous modulated accelerated radiotherapy combined with cisplatin concurrent chemotherapy: long-term results of a phase 2 study. Cancer. 2011; 117: 1874-83.

5. Xu ZX, Lin ZX, Fang JY, Wu KS, Du PL, Zeng Y, et al. Mortality Characteristic and Prediction of Nasopharyngeal Carcinoma in China from 1991 to 2013. Asian Pacific journal of cancer prevention : APJCP. 2015; 16: 6729-34.

6. Lee N, Harris J, Garden AS, Straube W, Glisson B, Xia P, et al. Intensity-modulated radiation therapy with or without chemotherapy for nasopharyngeal carcinoma: radiation therapy oncology group phase II trial 0225. Journal of clinical oncology : official journal of the American Society of Clinical Oncology. 2009; 27: 3684-90.

7. Sun X, Su S, Chen C, Han F, Zhao C, Xiao W, et al. Long-term outcomes of intensity-modulated radiotherapy for 868 patients with nasopharyngeal carcinoma: an analysis of survival and treatment toxicities. Radiotherapy and oncology : journal of the European Society for Therapeutic Radiology and Oncology. 2014; 110: 398-403.

8. Han F, Zhao C, Huang SM, Lu LX, Huang Y, Deng XW, et al. Long-term outcomes and prognostic factors of re-irradiation for locally recurrent nasopharyngeal carcinoma using intensity-modulated radiotherapy. Clinical oncology (Royal College of Radiologists (Great Britain)). 2012; 24: 569-76.

9. Kam MK, Teo PM, Chau RM, Cheung KY, Choi PH, Kwan WH, et al. Treatment of nasopharyngeal carcinoma with intensity-modulated radiotherapy: the Hong Kong experience. International journal of radiation oncology, biology, physics. 2004; 60: 1440-50.

10. Au JS, Law CK, Foo W, Lau WH. In-depth evaluation of the AJCC/UICC 1997 staging system of nasopharyngeal carcinoma: prognostic homogeneity and proposed refinements. International journal of radiation oncology, biology, physics. 2003; 56: 413-26.

11. Balkwill F, Mantovani A. Inflammation and cancer: back to Virchow? Lancet (London, England). 2001; 357: 539-45.

12. Chew HK, Wun $T$, Harvey D, Zhou H, White RH. Incidence of venous thromboembolism and its effect on survival among patients with common cancers. Archives of internal medicine. 2006; 166: 458-64.

13. Connolly GC, Francis CW. Cancer-associated thrombosis. Hematology / the Education Program of the American Society of Hematology American Society of Hematology Education Program. 2013; 2013: 684-91.

14. Koenig W. Fibrin(ogen) in cardiovascular disease: an update. Thrombosis and haemostasis. 2003; 89: 601-9.

15. Collen D, Tytgat GN, Claeys H, Piessens R. Metabolism and distribution of fibrinogen. I. Fibrinogen turnover in physiological conditions in humans. British journal of haematology. 1972; 22: 681-700.

16. Sun ZQ, Han XN, Wang HJ, Tang Y, Zhao ZL, Qu YL, et al. Prognostic significance of preoperative fibrinogen in patients with colon cancer. World journal of gastroenterology. 2014; 20: 8583-91.

17. Guo Q, Zhang B, Dong X, Xie Q, Guo E, Huang H, et al. Elevated levels of plasma fibrinogen in patients with pancreatic cancer: possible role of a distant metastasis predictor. Pancreas. 2009; 38: e75-9.

18. Pichler M, Hutterer GC, Stojakovic T, Mannweiler S, Pummer K, Zigeuner R. High plasma fibrinogen level represents an independent negative prognostic factor regarding cancer-specific, metastasis-free, as well as overall survival in a European cohort of non-metastatic renal cell carcinoma patients. British journal of cancer. 2013; 109: 1123-9.

19. Gooi Z, Fakhry C, Goldenberg D, Richmon J, Kiess AP. AHNS Series: Do you know your guidelines?Principles of radiation therapy for head and neck cancer: A review of the National Comprehensive Cancer Network guidelines. Head \& neck. 2016; 38: 987-92.

20. Chen A, Lee N, Yang C, Liu T, Narayan S, Vijayakumar S, et al. Comparison of intensity-modulated radiotherapy using helical tomotherapy and segmental multileaf collimator-based techniques for nasopharyngeal carcinoma: dosimetric analysis incorporating quality assurance guidelines from RTOG 0225. Technology in cancer research \& treatment. 2010; 9: 291-8.

21. Lan M, Chen C, Huang Y, Mao M, Han F, Liao J, et al. Elevated plasma fibrinogen level shows superior prognostic value than Epstein-Barr virus
DNA load for stage IVA/B nasopharyngeal carcinoma patients in the intensity-modulated radiotherapy era. Oncotarget. 2016.

22. Leung SF, Chan AT, Zee B, Ma B, Chan LY, Johnson PJ, et al. Pretherapy quantitative measurement of circulating Epstein-Barr virus DNA is predictive of posttherapy distant failure in patients with early-stage nasopharyngeal carcinoma of undifferentiated type. Cancer. 2003; 98: 288-91.

23. Tang LQ, Chen QY, Guo SS, Chen WH, Li CF, Zhang L, et al. The impact of plasma Epstein-Barr virus DNA and fibrinogen on nasopharyngeal carcinoma prognosis: an observational study. British journal of cancer. 2014; 111: 1102-11.

24. Takeuchi $\mathrm{H}$, Ikeuchi S, Kitagawa $\mathrm{Y}$, Shimada A, Oishi $\mathrm{T}$, Isobe $\mathrm{Y}$, et al. Pretreatment plasma fibrinogen level correlates with tumor progression and metastasis in patients with squamous cell carcinoma of the esophagus. Journal of gastroenterology and hepatology. 2007; 22: 2222-7.

25. Polterauer S, Seebacher V, Hefler-Frischmuth K, Grimm C, Heinze G, Tempfer $\mathrm{C}$, et al. Fibrinogen plasma levels are an independent prognostic parameter in patients with cervical cancer. American journal of obstetrics and gynecology. 2009; 200: 647.e1-7.

26. Gao J, Zhang HY, Xia YF. Increased platelet count is an indicator of metastasis in patients with nasopharyngeal carcinoma. Tumour biology : the journal of the International Society for Oncodevelopmental Biology and Medicine. 2013; 34: 39-45.

27. Kerlin B, Cooley BC, Isermann BH, Hernandez I, Sood R, Zogg M, et al. Cause-effect relation between hyperfibrinogenemia and vascular disease. Blood. 2004; 103: 1728-34.

28. Maragoudakis ME, Tsopanoglou NE, Andriopoulou P. Mechanism of thrombin-induced angiogenesis. Biochemical Society transactions. 2002; 30: 173-7.

29. Palumbo JS, Kombrinck KW, Drew AF, Grimes TS, Kiser JH, Degen JL, et al. Fibrinogen is an important determinant of the metastatic potential of circulating tumor cells. Blood. 2000; 96: 3302-9.

30. Camerer E, Oazi AA, Duong DN, Cornelissen I, Advincula R, Coughlin SR. Platelets, protease-activated receptors, and fibrinogen in hematogenous metastasis. Blood. 2004; 104: 397-401.

31. Wojtukiewicz MZ, Sierko E, Klement P, Rak J. The hemostatic system and angiogenesis in malignancy. Neoplasia (New York, NY). 2001; 3: 371-84.

32. Verheul HM, van Erp K, Homs MY, Yoon GS, van der Groep P, Rogers C, et al. The relationship of vascular endothelial growth factor and coagulation factor (fibrin and fibrinogen) expression in clear cell renal cell carcinoma. Urology. 2010; 75: 608-14.

33. Meadows KL, Hurwitz HI. Anti-VEGF therapies in the clinic. Cold Spring Harbor perspectives in medicine. 2012; 2.

34. Bensouda Y, Kaikani W, Ahbeddou N, Rahhali R, Jabri M, Mrabti H, et al. Treatment for metastatic nasopharyngeal carcinoma. European annals of otorhinolaryngology, head and neck diseases. 2011; 128: 79-85. 